Vercher-Ferrandis, M. (2021). La tutorización proactiva como factor de mejora en los resultados de la formación online. Revista de Investigación Educativa, 39(1), 91-109.

DOI: http://dx.doi.org/10.6018/rie.413901

\title{
La tutorización proactiva como factor de mejora en los resultados de la formación online
}

\section{Proactive tutoring as a factor in improving the results of online training}

Marisa Vercher-Ferrándiz

Universitat Politècnica de València

\begin{abstract}
Resumen
En el presente trabajo de investigación se plantea como hipótesis que un modelo de tutorización proactiva, centrado en el acompañamiento y seguimiento continuo del estudiante online, mejora la tasa de aprobados y la satisfacción de los estudiantes. Para validar dicha hipótesis se describen y comparan los resultados de las encuestas de satisfacción y la tasa de aprobados de los cursos impartidos a través del Plan de Estudios Coordinados en Asesoría Financiera del Centro de Formación Permanente de la Universitat Politècnica de València, incluyéndose en el estudio 27 cursos que siguen un modelo de tutorización proactiva con un total de 5.613 estudiantes matriculados y una muestra de 2.500 cuestionarios y 30 cursos que siguen un modelo de tutorización reactiva con un total de 1.862 estudiantes matriculados y una muestra de 583 cuestionarios. Los resultados obtenidos permiten aceptar la hipótesis de partida confirmando que en los cursos que se ha seguido un modelo de tutorización proactiva la tasa de aprobados es un 30\% más alta, y al respecto de la satisfacción del alumnado, en ambos tipos de tutorización, el patrón general de respuestas a las encuestas, con ciertas diferencias en la distribución de las mismas, es similar si bien, la satisfacción es más alta en los cursos que han seguido un modelo de tutorización proactivo, mientras que la insatisfacción es mayor en los de tutorización reactiva.

Palabras clave: Sistema tutorial; enseñanza a distancia; educación de adultos; formación continua; calidad de la enseñanza.
\end{abstract}

Correspondencia: Marisa Vercher-Ferrándiz, maverfer@upv.es, Departamento de Economía y Ciencias Sociales, Universitat Politècnica de Valéncia, 46022 València 


\begin{abstract}
This article establishes the hypothesis that a proactive tutoring model focused on the continuous monitoring and follow-up of online students increases success rates and student satisfaction. To validate this hypothesis, we describe and compare results from satisfaction surveys and the pass rate of a series of courses taught as part of the Coordinated Study Plan in Financial Advisory of the Permanent Training Center of the Polytechnic University of Valencia. This research considers 27 courses that follow a proactive tutoring model with a total of 5.613 enrolled students and a sample of 2.500 questionnaires, and 30 courses that follow a reactive tutoring model with a total of 1.139 enrolled students with a sample of 583 questionnaires. The results obtained corroborate the initial hypothesis, confirming that, in those courses that follow a proactive tutoring model, the success rate is 30\% higher. Regarding student satisfaction, both tutoring models show a similar response pattern to the surveys, with some differences in response distribution. However, student satisfaction is higher in those courses that follow a proactive tutoring model, while dissatisfaction is greater in those with a reactive tutoring one.

Key words: tutorial system; distance education; adult education; continuing training; quality of education.
\end{abstract}

\title{
Introducción
}

El aprendizaje a largo de la vida es la base para aumentar el conocimiento y las competencias de las personas en el entono profesional y en la vida en general (UNESCO, 2015) siendo la formación online uno de los modelos que más se está desarrollando dada la flexibilidad temporal y espacial, la compatibilidad con la vida profesional y personal de los individuos, la reducción de costes y el importante desarrollo de las TIC (Fernández-Robles y Cabero-Almenara, 2016), pero no solo en el entorno empresarial sino también en la educación superior, si bien, se requiere imponer una cultura de calidad siendo una estrategia efectiva para asegurarla, la selección de los docentes y tutores, su desarrollo profesional y una adecuada planificación de los servicios de apoyo y tutorización de los estudiantes, entre otros factores (Rovai y Downey, 2010).

La tutorización y apoyo a los estudiantes se presenta como uno de los factores de éxito o fracaso de los sistemas de formación a distancia (Sherry, 1995)technology selection and adoption, design issues, methods and strategies to increase interactivity and active learning, learner characteristics, learner support, operational issues, policy and management issues, equity and accessibility, and cost/benefit tradoffs. It is intended as a companion piece to Sherry and Morse's (1995, por lo que la labor tutorial integral durante el proceso de enseñanza-aprendizaje es uno de los criterios de calidad a tener en cuenta (Seoane-Pardo, García-Peñalvo, Nieto-Bosom, Fernández-Recio y Hernández-Tovar, 2006), y más atendiendo a que la población adulta es la principal receptora de la formación continua online, que por sus características particulares, requiere de ciertos principios metodológicos así como, de un flujo continuo de comunicación tutor-alumno al respecto de las reglas a seguir y de los procedimientos a utilizar y también, de atención individualizada a través de retroalimentación constante sobre el proceso de aprendizaje (Rodríguez Fernández, 
2014) y de facilitación del mismo además de anticiparse a las dificultades esperadas (Castañeda, Gutiérrez-Porlán, Prendes-Espinosa, y Sánchez-Vera, 2017).

Es frecuente en los estudiantes, tanto de formación presencial como de formación a distancia, que disminuya su motivación al tener que enfrentarse a obstáculos que requieren de su esfuerzo o a distracciones internas o externas que propician el posponer el inicio del estudio o el tiempo de dedicación (Broc Cavero, 2011), siendo la falta de estrategias, habilidades, destrezas y actitudes básicas para la formación online uno de los problemas más relevantes (Borges, 2005). Otro de los problemas que se presenta en el e-learning, comparado con otras modalidades de formación, es la alta tasa de abandono que se incrementa en los cursos cuyos participantes son trabajadores (FernándezJiménez, Mena-Rodríguez y Tójar-Hurtado, 2017)el e-learning ha sufrido una evolución constante. Ello se ha debido en parte a que es una nueva forma de aprendizaje adaptado a las necesidades de una sociedad cambiante, en la que los trabajadores necesitan capacitarse fuera de su horario laboral. De esta manera, la investigación en los procesos implícitos en el e-learning se hace imprescindible de cara a la mejora de la calidad de esta modalidad de formación. Por ello el objetivo de esta investigación se dirigió a 1 .

Son numerosas las investigaciones que se han centrado en estudiar las funciones y roles de los tutores de formación online. Para Cabero-Almenara (2004) y Llorente-Cejudo (2006), las funciones que realiza un tutor online son la función técnica al respecto de la utilización, dominio y trasmisión de conocimientos sobre el medio tecnológico utilizado, la función organizativa en cuanto a la acción formativa se refiere, la función académica en cuanto a diseño, atención de dudas y evaluación del aprendizaje, la función social al respecto de la integración de los estudiantes, dinamización y motivación, y por último, la función orientadora relacionada con la guía y asesoramiento en el proceso de aprendizaje, el ritmo de trabajo y la consecución de objetivos. En un estudio, realizado por Fernández-Jiménez et al. (2017)el e-learning ha sufrido una evolución constante. Ello se ha debido en parte a que es una nueva forma de aprendizaje adaptado a las necesidades de una sociedad cambiante, en la que los trabajadores necesitan capacitarse fuera de su horario laboral. De esta manera, la investigación en los procesos implícitos en el e-learning se hace imprescindible de cara a la mejora de la calidad de esta modalidad de formación. Por ello el objetivo de esta investigación se dirigió a 1, las funciones más valoradas por los estudiantes son por orden de importancia: la función organizativa, la académica y la orientadora, frente a la función técnica y la social. Aunque, no siempre el mismo profesional aborda todas las funciones, en algunos casos, los distintos roles son asumidos por varios profesionales que se encargan de los diferentes aspectos de la tutorización (Fernández Navas, 2016).

Pagano (2008), y al respecto de la función orientadora, expone que debe aplicarse a todos los estudiantes tutelados, estar dirigida a todas las dimensiones de la persona y llevarse a cabo durante todo el proceso, buscando la oportunidad y adecuándose a los momentos críticos del curso. La mediación, estimulación y aliento constante para evitar la desmotivación y la creación de un clima de trabajo confortable a través de la empatía es otra de las funciones que contribuyen a incrementar el compromiso de los estudiantes (Seoane-Pardo, 2014) y la base de todo ello es la interacción alumno-tutor para motivarlo y que mantenga el interés en aprender, además de evaluar la adquisición de conocimientos con el propósito de mejorar su aprendizaje (Franco Moreno, 2017). 
El establecimiento de un procedimiento tutorial, adecuado al modelo instruccional y a los plazos y entregas establecidos, puede ser un factor que ayude a los tutores a abordar su trabajo de manera eficiente. En el procedimiento se definen las pautas generales a seguir tales como: los plazos máximos de respuesta a los alumnos y la estructura de comunicaciones generales y particulares. Las comunicaciones generales suelen ser: la bienvenida al curso, la presentación del equipo docente, el envío de instrucciones técnicas y de la guía pedagógica, la comunicación de la liberación de contenidos y de los plazos de entrega de tareas; mientras que las personales suelen ser: el envío de retroalimentación ante las entregas (López Martínez, 2009), el asesoramiento ante la evaluación final y por último la comunicación final de los resultados. Con todo ello se define un modelo tutorial reactivo en el que el tutor reacciona ante la solicitud de un alumno contestando a sus dudas y requerimientos y realiza las tareas y funciones definidas, según el protocolo establecido, lo que estandariza la interacción alumno-tutor.

En un estudio sobre buenas prácticas en la comunicación e interacción en la formación online, Vlachopoulos y Makri (2019) concluyen que los estudiantes interactúan con sus tutores cuando necesitan aclaraciones sobre: el contenido del curso, los requisitos de evaluación, los plazos de entrega e incluso, sobre el uso del sistema informático pero, no todos los tutores son igualmente exitosos en la promoción de interacciones significativas. Para estos autores, la clave se encuentra en la oportuna retroalimentación e inmediatez que los tutores deben proporcionar a sus alumnos. Además, los tutores deben de adoptar diversos roles como: el de facilitador, el de mentor y el de asesor. El objetivo final es focalizarse en alentar, motivar y mejorar el rendimiento de los estudiantes, dirigiendo su aprendizaje y ofreciéndoles un apoyo continuado.

Siguiendo con la exposición de Vlachopoulos y Makri, la interacción significativa entre tutor y alumno debería ser una prioridad para los tutores, para con ello, construir relaciones saludables a través del diálogo frecuente, mostrándose accesibles en todo momento, dirigiéndose a ellos de manera informal y haciendo preguntas sobre su bienestar pues, la interacción alumno-tutor, produce una sensación de conectividad reduciendo la distancia.

En un modelo de tutorización proactiva, el tutor, además de seguir el protocolo de comunicaciones establecido, trata de generar una relación significativa con sus estudiantes a través de su seguimiento personalizado, contactándoles por iniciativa propia, sin esperar a que le requieran, mostrando preocupación si demoran el inicio del curso y no acceden con regularidad, o bien, no cumplen con los plazos de las entregas, ofreciéndoles entonces su ayuda, además de consejos sobre técnicas de estudio, tutorías personalizadas sobre las dificultades que se van encontrando, guía y apoyo durante todo el proceso (Vercher-Ferrándiz, 2019).

De la misma forma y en el caso de que el progreso del estudiante sea el adecuado, el tutor le contacta igualmente pero, en este caso, para felicitarle y reconocerle el esfuerzo y dedicación que está realizando, con el objetivo de que continúe motivado y focalizado en su avance y aprendizaje, interesándose también, tanto en la faceta académica como en la personal, poniéndose a su disposición y propiciando interacciones significativas (Vercher-Ferrándiz, 2019). 
Los modelos de tutorización proactiva implican el seguimiento y acompañamiento exhaustivo de los estudiantes, lo que supone una mayor dedicación y esfuerzo por parte del tutor, así como compromiso, responsabilidad y disciplina (Sagastume, Morales, Amado y Hernández, 2018) y el desarrollo de competencias en inteligencia emocional tales como: la empatía, la orientación al servicio y la comunicación, entre otras (Andrew Youde, 2016).

\section{Método}

\section{Objetivos}

Se plantea como propósito de esta investigación validar la hipótesis de que un modelo de tutorización personalizado y proactivo, centrado en el acompañamiento y seguimiento continuo de los estudiantes, mejora los resultados de aprendizaje, en cuanto a número de aptos se refiere tras el proceso de evaluación final y el nivel de satisfacción percibido de los participantes.

Para ello, se describen y comparan: los resultados de las encuestas de satisfacción, la tasa de respuesta y el porcentaje de aptos en base al modelo tutorial seguido de los programas formativos online impartidos a través del Plan de Estudios Coordinados en Asesoría Financiera del Centro de Formación Permanente (CFP) de la Universitat Politècnica de València (UPV).

\section{Población y Muestra}

La población objeto de estudio son los cursos de formación continua del CFP de la Universitat Politècnica de València impartidos por el equipo de docentes del Plan de Estudios Coordinados en Asesoría Financiera en la modalidad online en el periodo de 2011 a 2018.

Del universo total de cursos, se seleccionan aquellos que han finalizado y que disponen de la encuesta de calidad online facilitada por CFP al término del curso. Se desestiman aquellos que no han finalizado o no tienen respuestas a la encuesta. La muestra resultante es de 27 cursos que siguen un modelo de tutorización proactiva con un total de 5.613 estudiantes matriculados y una muestra de 2.500 cuestionarios y 30 cursos que siguen un modelo de tutorización reactiva con un total de 1.139 estudiantes matriculados y una muestra de 583 cuestionarios.

\section{Instrumento}

De forma general, el Centro de Formación Permanente facilita a los estudiantes, al terminar el curso, un modelo de encuesta breve online que consta de tres ítems (ver Tabla 1) relacionados con: a) la labor de tutor, b) la organización general del curso y c) la satisfacción del estudiante ante sus expectativas hacia el curso. El estudiante debe valorar cada afirmación según una escala tipo Likert, con un rango de 5 puntos que van desde "Totalmente en desacuerdo" (1), "Más bien en desacuerdo" (2) “Término medio" (3), "Más bien de acuerdo" (4) y "Totalmente de acuerdo" (5) o bien, "Sin información suficiente" ( )." 
Tabla 1

Modelo de encuesta

\section{Encuesta breve}

\begin{tabular}{ll}
\hline Tutor & $\begin{array}{l}\text { A. El profesor/es o tutor/es ha/n cubierto mis expectativas, facilitando el apre- } \\
\text { ndizaje (dominio de la materia, resolución de dudas, atención al alumno, etc.) }\end{array}$ \\
$\begin{array}{l}\text { Orga- } \\
\text { nización }\end{array}$ & $\begin{array}{l}\text { B. La organización general y calidad del curso han sido buenos (materiales, } \\
\text { contenidos, duración del curso, infraestructura material o tecnológica, etc.) }\end{array}$ \\
Satisfacción & C. Mis expectativas ante el curso han quedado satisfechas \\
\hline
\end{tabular}

El índice de confiabilidad Alfa de Cronbach del instrumento es de $\alpha=00889$, por lo que se puede afirmar que al ser $\alpha>0.8$ el instrumento es altamente confiable, tal y como expone Bryman y Cramer (1990: 71) citados por Cohen, Manion, y Morrison (2017).

\section{Procedimiento de recogida y análisis de datos}

Las respuestas de los participantes se exportan directamente de la plataforma de gestión del Centro de Formación Permanente de la UPV en un archivo de Microsoft Excel con el fin de importarlo al programa MAXQDA Analytics Pro 2020 y a SPSS 22.0, se tabulan los datos organizándose la información en columnas siendo éstas: Tutor, Organización y Satisfacción, definiéndose además, la variable instrumental Modelo que identifica el modelo de tutorización que sigue cada uno de los cursos y la variable instrumental Duración del curso, lo que permitirá comparar los cuestionarios a partir de estas nuevas variables.

$\mathrm{Al}$ respecto del tratamiento de las respuestas de los participantes a las encuestas, se identifica cada respuesta con el identificador del curso compuesto por la letra R, en el caso de cursos de tutorización reactiva que son aquellos en los que el tutor se limita a seguir el protocolo de tutorización establecido siendo éste el envío de comunicaciones estandarizadas y la atención a las consultas de los estudiantes en tiempo y forma; y con la letra P los cursos que siguen un modelo de tutorización proactiva en los que el tutor además de seguir el protocolo de comunicaciones establecido, está pendiente del avance, seguimiento y acompañamiento del alumno, contactando con él, por iniciativa propia, para mostrar su preocupación por un avance deficiente, ofreciéndole su ayuda o bien felicitándolo por su adecuado progreso para que siga motivado y focalizado en su aprendizaje. Se incluye en el identificador de cada respuesta una letra que identifica la duración del curso siendo: A) menos de 5 ECTS, B) entre 5 y 9 ECTS, C) entre 10 y 14 ECTS y D) 15 o más ECTS y por último un número secuencial y único para cada respuesta. En total se importan 2.500 cuestionarios pertenecientes a los cursos que han seguido un modelo de tutorización proactiva y 583 pertenecientes a los de tutorización reactiva. 


\section{Resultados}

Para explorar y describir las relaciones entre el modelo tutorial y las frecuencias de respuestas a las encuestas en lo que respecta a la variable Tutor, se analiza la distribución de los datos (Tabla 2) siendo el ítem del cuestionario al que deben responder los estudiantes: "El profesor/es o tutor/es ha/n cubierto mis expectativas, facilitando el aprendizaje (dominio de la materia, resolución de dudas, atención al alumno, etc.)".

En lo que respecta a la labor del tutor entre los dos modelos de tutorización seguidos, se aprecia que la proporción de alumnos que contestan "Más bien en desacuerdo" (2) y "Término medio" (3) es mayor en aquellos cursos que siguen un modelo tutorial reactivo, mientras que la proporción de alumnos que contesta "Más bien de acuerdo" (4) es mayor en los cursos que siguen un modelo tutorial proactivo, siendo la diferencia entre las repuestas extremas inferiores a un punto porcentual. Se reproduce en ambos tipos de tutorización una distribución asimétrica de los datos produciéndose mayores diferencias en las puntuaciones centrales de la escala.

Tabla 2

Tabla cruzada modelo tutorial - Variable Tutor

\begin{tabular}{ccccccc}
\hline Modelo & $\begin{array}{c}\text { Totalmente en } \\
\text { desacuerdo }\end{array}$ & $\begin{array}{c}\text { Más bien en } \\
\text { desacuerdo }\end{array}$ & $\begin{array}{c}\text { Término } \\
\text { medio }\end{array}$ & $\begin{array}{c}\text { Más bien } \\
\text { de acuerdo }\end{array}$ & $\begin{array}{c}\text { Totalmente } \\
\text { de acuerdo }\end{array}$ & Total \\
\hline \multirow{2}{*}{ Proactivo } & $1.5 \%$ & $1.8 \%$ & $12.7 \%$ & $38.9 \%$ & $45.1 \%$ & $100 \%$ \\
& $(31)$ & $(38)$ & $(265)$ & $(808)$ & $(937)$ & $(2079)$ \\
\hline \multirow{2}{*}{ Reactivo } & $2.2 \%$ & $5.3 \%$ & $16.9 \%$ & $30.8 \%$ & $44.8 \%$ & $100 \%$ \\
& $(9)$ & $(22)$ & $(70)$ & $(127)$ & $(185)$ & $(413)$ \\
\hline \multirow{2}{*}{ Total } & $1.6 \%$ & $2.4 \%$ & $13.4 \%$ & $37.5 \%$ & $45.0 \%$ & $100 \%$ \\
& $(40)$ & $(60)$ & $(335)$ & $(935)$ & $(1122)$ & $(2492)$ \\
\hline
\end{tabular}

Se puede observar, al respecto de la variable Tutor (Tabla 3), que en los cursos que siguen un modelo tutorial proactivo, la media es más alta y menor la desviación típica, así como, también es menor el error y el porcentaje de respuestas en blanco.

Tabla 3

Descriptivos variable Tutor por modelo tutorial

\begin{tabular}{lcccccc}
\hline Modelo & Media & $\begin{array}{c}\text { Des. Típica } \\
\text { (muestra) }\end{array}$ & $\begin{array}{c}\text { Error } \\
\text { típico }\end{array}$ & Mediana & Rango & $\begin{array}{c}\text { Perdidos } \\
\text { (\%) }\end{array}$ \\
\hline Proactivo & 4.24 & .853 & .02 & 4 & 4 & 16.84 \\
Reactivo & 4.11 & 1.008 & .05 & 4 & 4 & 29.16 \\
\hline
\end{tabular}

Se aplica la prueba de Mann-Whitney para determinar si hay una diferencia estadísticamente significativa al respecto de la variable Tutor entre un modelo tutorial y 
otro, $(\mathrm{U}=407013.000, \mathrm{p}=0.071)$, dado que $\mathrm{p}>0.05$ se concluye que la diferencia no es estadísticamente significativa.

Se analiza la distribución de los datos en las encuestas al respecto de la variable Organización (Tabla 4), siendo el ítem del cuestionario al que deben de responder los estudiantes: "La organización general y calidad del curso han sido buenos (materiales, contenidos, duración del curso, infraestructura material o tecnológica, etc.)".

En lo que respecta a la variable Organización entre los dos modelos de tutorización seguidos, se aprecia que la proporción de alumnos que indicaron "Más bien en desacuerdo" (2) y "Término medio" (3) fue mayor en los de cursos de tutorización reactiva que en los de tutorización proactiva, cuestión esta que se invierte en las respuestas "Más bien de acuerdo" (4) y "Totalmente de acuerdo" (5).

Tabla 4

Tabla cruzada modelo tutorial - Variable Organización

\begin{tabular}{ccccccc} 
Modelo & $\begin{array}{c}\text { Totalmente en } \\
\text { desacuerdo }\end{array}$ & $\begin{array}{c}\text { Más bien en } \\
\text { desacuerdo }\end{array}$ & $\begin{array}{c}\text { Término } \\
\text { medio }\end{array}$ & $\begin{array}{c}\text { Más bien } \\
\text { de acuerdo }\end{array}$ & $\begin{array}{c}\text { Totalmente } \\
\text { de acuerdo }\end{array}$ & Total \\
\hline \multirow{2}{*}{ Proactivo } & $1.5 \%$ & $2.9 \%$ & $11.7 \%$ & $41.7 \%$ & $42.3 \%$ & $100 \%$ \\
& $(33)$ & $(66)$ & $(264)$ & $(945)$ & $(958)$ & $(2266)$ \\
\hline \multirow{2}{*}{ Reactivo } & $1.1 \%$ & $6.0 \%$ & $17.1 \%$ & $35.0 \%$ & $40.9 \%$ & $100 \%$ \\
& $(5)$ & $(28)$ & $(80)$ & $(164)$ & $(192)$ & $(469)$ \\
\hline \multirow{2}{*}{ Total } & $1.4 \%$ & $3.4 \%$ & $12.6 \%$ & $40.5 \%$ & $42.0 \%$ & $100 \%$ \\
& $(38)$ & $(94)$ & $(344)$ & $(1109)$ & $(1150)$ & $(2735)$ \\
\hline
\end{tabular}

Se puede observar, al respecto de la variable Organización (Tabla 5), que en los cursos que siguen un modelo tutorial proactivo, la media es más alta y menor la desviación típica, así como, también es menor el error y el porcentaje de respuestas en blanco en los primeros que en los segundos.

Tabla 5

Descriptivos variable Organización por modelo tutorial

\begin{tabular}{lcccccc}
\hline Modelo & Media & $\begin{array}{c}\text { Des. Típica } \\
\text { (muestra) }\end{array}$ & $\begin{array}{c}\text { Error } \\
\text { típico }\end{array}$ & Mediana & Rango & $\begin{array}{c}\text { Perdidos } \\
\text { (\%) }\end{array}$ \\
\hline Proactivo & 4.20 & .863 & .02 & 4 & 4 & 9.36 \\
Reactivo & 4.09 & .952 & .04 & 4 & 4 & 19.55 \\
\hline
\end{tabular}

Se aplica la prueba de Mann-Whitney para determinar si hay una diferencia estadísticamente significativa al respecto de la variable Organización, entre un modelo tutorial 
y otro, $(U=500536.500, p=0.032)$, dado que $p<0.05$ se concluye que la diferencia es estadísticamente significativa.

Se analiza la distribución de los datos en las encuestas al respecto de la variable Satisfacción (Tabla 6), en función del modelo tutorial seguido, siendo el ítem del cuestionario al que deben de responder los estudiantes: "Mis expectativas ante el curso han quedado satisfechas".

Se puede observar que, las respuesta positivas, "Más bien de acuerdo" (4) y "Totalmente de acuerdo" (5), son más frecuentes en los cursos que siguen un modelo de tutorización proactiva, con una diferencia de 6.2 puntos porcentuales en la primera y 2.1 en la segunda. Así mismo, las opiniones negativas o neutras, "Totalmente en desacuerdo" (1), "Más bien en desacuerdo" (2) y "Término medio" (3), son más frecuentes en los cursos de tutorización reactiva.

Tabla 6

Tabla cruzada modelo tutorial - Variable Satisfacción

\begin{tabular}{ccccccc}
\hline Modelo & $\begin{array}{c}\text { Totalmente en } \\
\text { desacuerdo }\end{array}$ & $\begin{array}{c}\text { Más bien en } \\
\text { desacuerdo }\end{array}$ & $\begin{array}{c}\text { Término } \\
\text { medio }\end{array}$ & $\begin{array}{c}\text { Más bien } \\
\text { de acuerdo }\end{array}$ & $\begin{array}{c}\text { Totalmente } \\
\text { de acuerdo }\end{array}$ & Total \\
\hline Proactivo & $1.7 \%$ & $3.0 \%$ & $12.2 \%$ & $40.7 \%$ & $42.4 \%$ & $100 \%$ \\
& $(38)$ & $(67)$ & $(278)$ & $(925)$ & $(963)$ & $(2271)$ \\
\hline Reactivo & $2.1 \%$ & $6.2 \%$ & $16.7 \%$ & $34.5 \%$ & $40.3 \%$ & $100 \%$ \\
& $(10)$ & $(29)$ & $(78)$ & $(161)$ & $(188)$ & $(466)$ \\
\hline Total & $1.8 \%$ & $3.5 \%$ & $13.0 \%$ & $39.7 \%$ & $42.1 \%$ & $100 \%$ \\
& $(48)$ & $(96)$ & $(356)$ & $(1086)$ & $(1151)$ & $(2737)$ \\
\hline
\end{tabular}

Al respecto de esta variable, se comprueba en la Tabla 7 que, en los cursos que siguen un modelo tutorial proactivo la media es más alta y menor la desviación típica así como, también es menor el error y el porcentaje de respuestas en blanco.

Tabla 7

Descriptivos variable Satisfacción por modelo tutorial

\begin{tabular}{lcccccc}
\hline Modelo & Media & $\begin{array}{c}\text { Des. Típica } \\
\text { (muestra) }\end{array}$ & $\begin{array}{c}\text { Error } \\
\text { típico }\end{array}$ & Mediana & Rango & $\begin{array}{c}\text { Perdidos } \\
\text { (\%) }\end{array}$ \\
\hline Proactivo & 4.19 & .882 & .02 & .02 & 4 & $9.16 \%$ \\
Reactivo & 4.05 & 1.005 & .05 & .05 & 4 & $20.07 \%$ \\
\hline
\end{tabular}

Se aplica la prueba de Mann-Whitney para determinar si hay diferencia estadísticamente significativa al respecto de la variable Satisfacción entre un modelo tutorial y otro, $(\mathrm{U}=493847.000, \mathrm{p}=0.014)$ dado que $\mathrm{p}<0.05$ se concluye que la diferencia es estadísticamente significativa.

Se comparan los resultados de los cursos según el modelo tutorial seguido en lo que respecta al porcentaje de aptos y a la tasa de respuesta a las encuestas (Tabla 8). 
Tabla 8

Resultados aptos y respuesta según modelo de tutorización

\begin{tabular}{lcccc}
\hline Modelo Tutorización & No Cursos & No Matrículas & Aptos & $\begin{array}{c}\text { Respuesta } \\
\text { Encuesta }\end{array}$ \\
\hline Proactivo & 27 & 5613 & 5133 & 2500 \\
Reactivo & 30 & 1862 & 1139 & 583 \\
\hline
\end{tabular}

En base al número de matrículas, se desprende que, la tasa de aptos tras la evaluación de la acción formativa, consistente ésta en la realización, entrega y superación de determinadas actividades de evaluación continua y la superación de un examen final, es un 30\% más alta en los cursos que siguieron un modelo de tutorización proactiva que en los cursos que siguieron un modelo reactivo, siendo la tasa de respuesta a las encuesta un $14 \%$ más alta en los primeros que en los segundos (Figura 1).

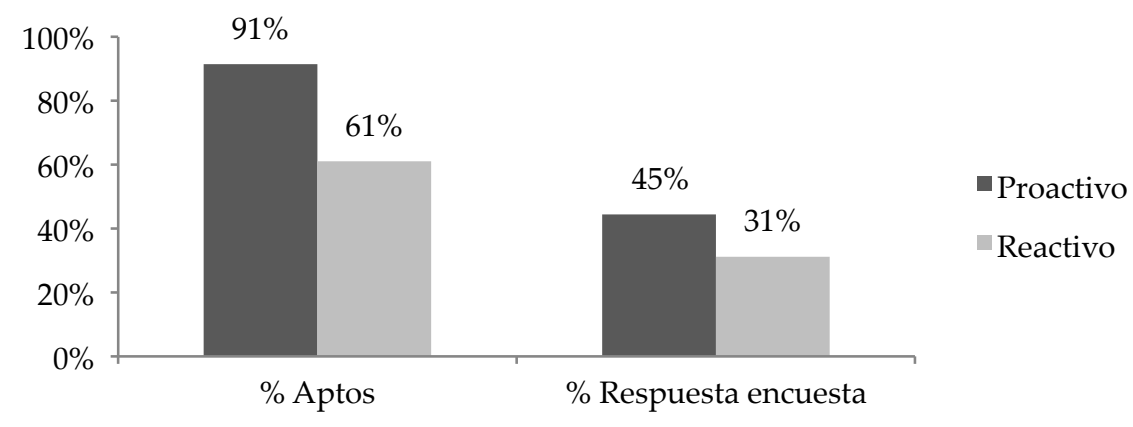

Figura 1. Comparativa tasa de aptos y respuesta a las encuestas según modelo tutorial.

Del análisis realizado, en base al modelo tutorial seguido, se puede concluir que existe una diferencia estadísticamente significativa en la distribución de las respuestas en cuanto a las variables Organización y Satisfacción, no siendo así en la variable Tutor, pudiéndose explicar esta cuestión en base a que son los mismos profesionales los que trabajan indistintamente en unos y otros cursos.

En cuanto al porcentaje de aprobados y la tasa de respuesta a las encuestas, es mayor en los cursos que han seguido un modelo de tutorización proactivo siendo además, menor el número de respuestas en blanco, que podría ser explicado en base a que, un modelo de tutorización proactiva, en el que el tutor está pendiente del avance del estudiante, dirigiéndolo, aconsejándolo y acompañándolo en todo momento, consigue mayor implicación y compromiso por parte del alumnado.

Se procede a realizar un análisis comparativo por modelo tutorial, atendiendo a la duración de los cursos, para explorar la influencia de esta variable en cuanto: al nivel de satisfacción de los estudiantes, el número de aptos y el número de respuestas a las encuestas. La duración de un curso está determinada por el número de ECTS que, influye en el número de semanas o meses que se requieren para finalizarlo. 
Se comparan visualmente las frecuencias de respuestas de la variable Satisfacción, en base a la duración de los cursos y el modelo tutorial seguido y con tal de facilitar el análisis, se agrupan las respuestas de los estudiantes que mostraron desacuerdo con la afirmación "Mis expectativas ante el curso han quedado satisfechas" al contestar: "Totalmente en desacuerdo" (1) o "Más bien en desacuerdo" (2); y las que mostraron acuerdo al contestar: "Más bien de acuerdo" (4) y "Totalmente de acuerdo" (5).

Se puede observar en la Figura 2 que, la diferencia mayor, entre los alumnos que estaban de acuerdo con que sus expectativas habían quedado satisfechas y en cuanto al modelo de tutorización seguido, se produce en los cursos de menos de 5 ECTS. Se observa también que, salvo en los de más de 15 ECTS, el nivel de satisfacción es mayor en los cursos proactivos que en los reactivos, con independencia de la duración del curso. En lo que respecta al desacuerdo con la afirmación, se evidencia una diferencia mayor los cursos más cortos.

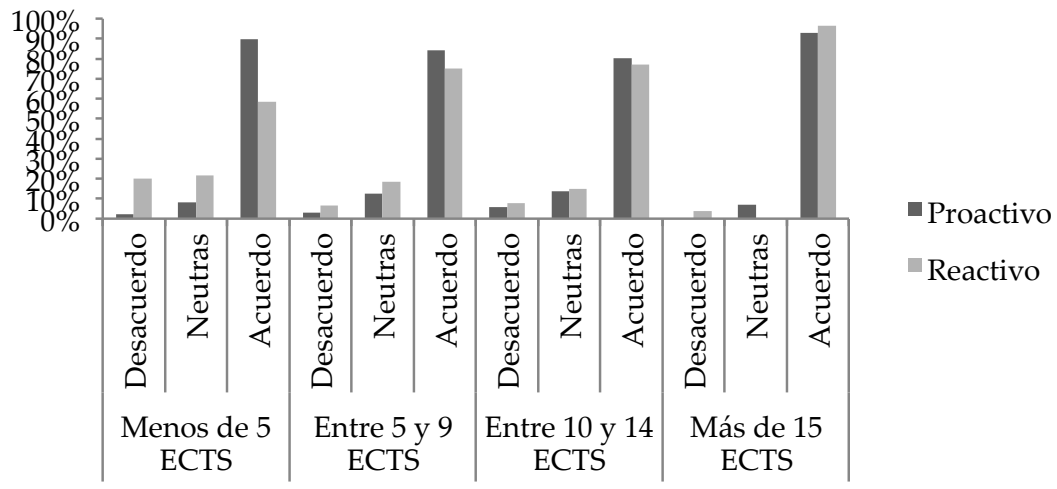

Figura 2. Modelo tutorial - Variable Satisfacción - Duración curso

Al comparar las medias de la variable Satisfacción en base al modelo tutorial seguido y la duración del curso, se comprueba (Tabla 9) que, éstas son mayores en los cursos más cortos que han seguido el modelo proactivo, se igualan en los cursos de 10 a 14 ECTS de duración y en los de mayor duración, la media es mayor en los que han seguido un modelo reactivo. En todos los cursos, con independencia de la duración, la desviación estándar es menor en los cursos que han seguido el modelo proactivo.

Tabla 9

Descriptivos - Modelo tutorización por duración - Variable Satisfacción

\begin{tabular}{lcccccc}
\hline & Proactivo & \multicolumn{3}{c}{ Reactivo } \\
\hline Duración & $\mathbf{N}$ & Media & Desv. Están. & $\mathbf{N}$ & Media & Desv. Están. \\
\hline Menos de 5 ECTS & 451 & 4.377 & .7629 & 60 & 3.650 & 1.2188 \\
Entre 5 y 9 ECTS & 205 & 4.268 & .8112 & 251 & 4.056 & .9364 \\
\hline
\end{tabular}




\begin{tabular}{lcccccc}
\hline & Proactivo & \multicolumn{3}{c}{ Reactivo } \\
\hline Duración & $\mathbf{N}$ & Media & Desv. Están. & $\mathbf{N}$ & Media & Desv. Están. \\
\hline Entre 10 y 14 ECTS & 1514 & 4.111 & .9267 & 127 & 4.110 & 1.0097 \\
15 o más ECTS & 101 & 4.436 & .6232 & 28 & 4.536 & .8381 \\
\hline
\end{tabular}

El resultado del test de Kruskall-Wallis revela que $(\chi 2=60.413$, g.l. $=7, p=0.000)$, y dado que $\mathrm{p}<0.05$ existe una diferencia estadísticamente significativa entre al menos un par de las combinaciones comparadas (Tabla 10).

Tabla 10

Modelo tutorización por duración - Variable Satisfacción - Prueba Kruskal-Wallis

\begin{tabular}{lcccc}
\hline & Proactivo & & Reactivo & \\
\hline Duración & $\mathbf{N}$ & Rango promedio & $\mathbf{N}$ & Rango promedio \\
\hline Menos de 5 ECTS & 451 & 1534.72 & 60 & 1048.48 \\
Entre 5 y 9 ECTS & 205 & 1436.86 & 251 & 1273.47 \\
Entre 10 y 14 ECTS & 1514 & 1321.13 & 127 & 1352.56 \\
15 o más ECTS & 101 & 1558.32 & 28 & 1726.14 \\
Total & 2271 & & 466 & \\
\hline
\end{tabular}

Para conocer específicamente entre qué pares de combinaciones se produjo una diferencia significativa $(p<0.05)$, se realizaron comparaciones post hoc mediante el test de Tukey, resultando significativas las que se muestran en la Tabla 11, en la que se observa que los únicos cursos cuya media de satisfacción difiere significativamente del resto de cursos, son los de 5 ECTS o menos que siguen un modelo tutorial reactivo (RA).

Tabla 11

HSD Tukey - Variable Satisfacción

\begin{tabular}{ccccccc}
\hline \multirow{2}{*}{$\begin{array}{c}\text { (I) Modelo- } \\
\text { Duración }\end{array}$} & $\begin{array}{c}\text { (J) Modelo- } \\
\text { Duración }\end{array}$ & $\begin{array}{c}\text { Diferencia } \\
\text { de medias } \\
\text { (I-J) }\end{array}$ & Error estándar & Sig. & \multicolumn{2}{c}{$\begin{array}{c}\text { 95\% de intervalo de } \\
\text { confianza }\end{array}$} \\
\hline PA & PC & $.2660^{*}$ & .0480 & .000 & .120 & .412 \\
inferior & $\begin{array}{c}\text { Límite } \\
\text { superior }\end{array}$ \\
& RB & $.3212^{*}$ & .0705 & .000 & .107 & .535 \\
PC & PD & $-.3247^{*}$ & .0920 & .010 & -.604 & -.046 \\
PD & RB & $.3799^{*}$ & .1055 & .008 & .060 & .700 \\
RA & PA & $-.7269^{*}$ & .1231 & .000 & -1.100 & -.354 \\
& PB & $-.6183^{*}$ & .1314 & .000 & -1.017 & -.220 \\
& PC & $-.4610^{*}$ & .1179 & .002 & -.819 & -.103 \\
\hline
\end{tabular}




\begin{tabular}{lcccccc}
\hline \multirow{2}{*}{$\begin{array}{l}\text { (I) Modelo- } \\
\text { Duración }\end{array}$} & $\begin{array}{c}\text { (J) Modelo- } \\
\text { Duración }\end{array}$ & $\begin{array}{c}\text { Diferencia } \\
\text { de medias } \\
\text { (I-J) }\end{array}$ & Error estándar & Sig. & \multicolumn{2}{c}{$\begin{array}{c}\text { 95\% de intervalo de } \\
\text { confianza }\end{array}$} \\
\cline { 5 - 7 } & & & & & $\begin{array}{c}\text { Límite } \\
\text { inferior }\end{array}$ & $\begin{array}{c}\text { Límite } \\
\text { superior }\end{array}$ \\
\hline PD & $-.7856^{*}$ & .1460 & .000 & -1.228 & -.343 \\
& RB & $-.4058^{*}$ & .1287 & .035 & -.796 & -.015 \\
& RC & $-.4602^{*}$ & .1403 & .023 & .120 & .412 \\
RD & $-.8857^{*}$ & .2050 & .000 & .107 & .535 \\
\hline
\end{tabular}

$\mathrm{Al}$ analizar los subconjuntos homogéneos resultantes de la prueba de Tukey (Tabla 12), es decir aquellos grupos dónde las medias no son estadísticamente diferentes entre sí, se puede observar que han emergido 3 subconjuntos, siendo los únicos cursos que difieren del resto los de 5 o menos ECTS que han seguido un modelo tutorial reactivo, por lo que se puede concluir que no hay una diferencia clara en el nivel de satisfacción del alumnado entre un modelo tutorial y otro al considerar la duración de los cursos.

Tabla 12

HSD Tukey a,b - Subconjuntos homogéneos - Variable Satisfacción

\begin{tabular}{lcccc}
\hline \multirow{2}{*}{ Modelo-Duración } & N & \multicolumn{3}{c}{ Subconjunto para alfa $=\mathbf{0 . 0 5}$} \\
& \multicolumn{5}{c}{1} & 2 & 3 \\
\hline REAC-menos de 5 ECTS & 60 & 3.650 & \\
REAC-entre 5 y 9 ECTS & 251 & & 4.056 & \\
REAC-entre 10 y 14 CTS & 127 & 4.110 & \\
PROAC-entre 10 y 14 CTS & 1514 & 4.111 & \\
PROAC-entre 5 y 9 ECTS & 205 & 4.268 & 4.268 \\
PROAC-menos de 5 ECTS & 451 & 4.377 & 4.377 \\
PROAC-más de 15 ECTS & 101 & 4.436 & 4.436 \\
REAC-más de 15 ECTS & 28 & & & 4.536 \\
Sig. & & 1.000 & .061 & .424 \\
\hline
\end{tabular}

a. Utiliza el tamaño de la muestra de la media armónica =97,685.

b. Los tamaños de grupo no son iguales. Se utiliza la media armónica de los tamaños de grupo.

Al comparar la tasa de aptos entre los modelos tutoriales seguidos en base a la duración de los cursos (Figura 3), se puede observar que la diferencia mayor (59\%), se produce en los de más de 15 ECTS, y la menor (12\%) en los de menos de 5 ECTS, pudiéndose explicar en base a la dificultad y extensión del programa de contenidos y el desgaste que supone para un alumno estudiar un largo periodo de tiempo a través de este medio sin el seguimiento y apoyo continuado del tutor. 


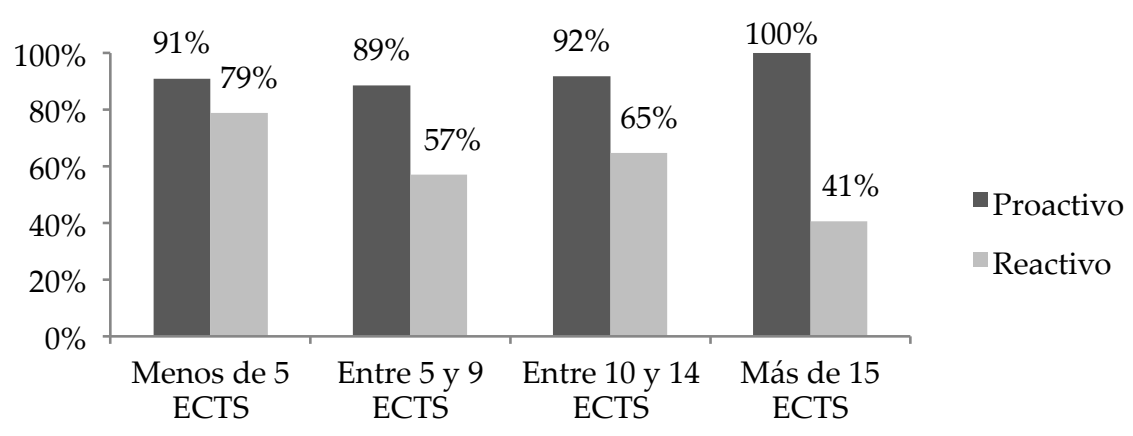

Figura 3. Cursos - Duración - Tasa de aptos

En cuanto a la tasa de respuesta a las encuestas, en la comparación destaca que, el porcentaje de respuesta es mayor en los cursos más largos, siendo la diferencia entre proactivos y reactivos de un $47 \%$, mientras que en los cursos entre 10 y 14 ECTS, la diferencia es mínima (Figura 4).

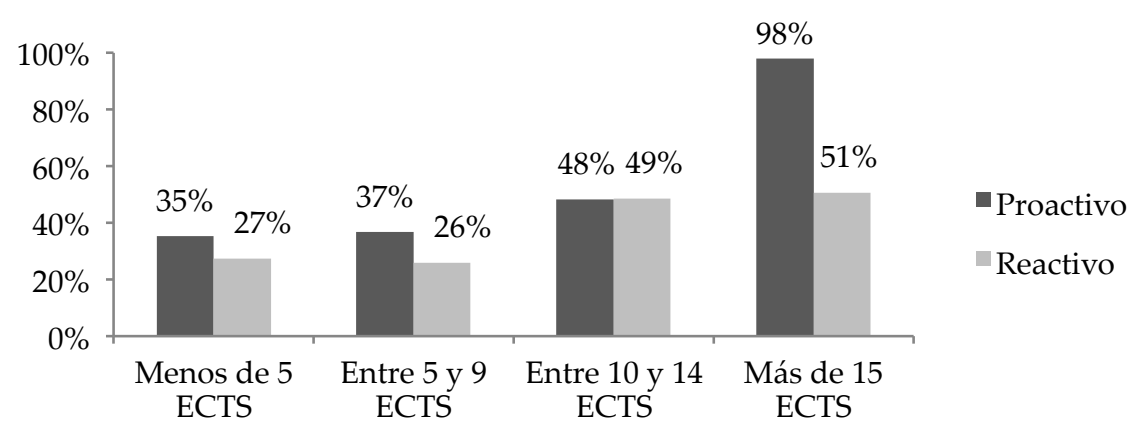

Figura 4. Cursos - Duración - Tasa de respuesta

En el análisis realizado en base al modelo tutorial seguido y la duración de los cursos, se puede concluir que, tan solo en los cursos de 5 o menos ECTS la media de la variable Satisfacción difiere significativamente del resto de cursos, encontrándose además 4 pares de combinaciones en las que la diferencia de medias también es significativa, si bien no hay un patrón claro en los subconjuntos homogéneos que han emergido, por lo que se concluye que la variable duración del curso no tiene una incidencia clara en el nivel de satisfacción del alumnado.

En lo que respeta a la tasa de aprobados entre un modelo tutorial y otro, en base a la duración de los cursos, la diferencia es mayor en los cursos de más duración y menor en los más cortos, siendo en todos los casos mayor en los cursos en los que se ha seguido un modelo tutorial proactivo, lo que pone de manifiesto la importancia del seguimiento y acompañamiento del tutor en los programas formativos de mayor complejidad y extensión. 
En cuanto a la tasa de respuesta, la mayor diferencia entre ambos modelos tutoriales, se encuentra en los cursos de 15 ECTS igualándose en los cursos entre 10 y 14 ECTS.

Queda demostrada la hipótesis inicial que afirmaba que un modelo de tutorización personalizado y proactivo, centrado en el acompañamiento y seguimiento continuo de los estudiantes, mejora los resultados de aprendizaje, en cuanto a la tasa de aptos se refiere y el nivel de satisfacción percibido de los participantes.

$\mathrm{Al}$ incluir en el estudio la variable Duración, se concluye que el modelo tutorial seguido tiene mayor relevancia en los cursos más extensos ya que las diferencias en la tasa de aptos y de respuesta son mayores pero no se presenta evidencia de que esta variable incida en el nivel de satisfacción del alumnado al no encontrarse diferencias significativas entre los dos modelos de tutorización y las duraciones de los cursos estudiados, no presentándose un patrón claro.

\section{Discusión y conclusiones}

El papel del tutor en la formación online emerge como uno de los factores de calidad del modelo formativo, siendo descrito como mediación docente y apoyo al alumnado (Sherry, 1995)technology selection and adoption, design issues, methods and strategies to increase interactivity and active learning, learner characteristics, learner support, operational issues, policy and management issues, equity and accessibility, and cost/benefit tradoffs. It is intended as a companion piece to Sherry and Morse's (1995, estando basado éste en una relación orientadora (Martínez y Briones, 2007) en la que el tutor o tutores ejercen una labor tutorial integral durante todo el proceso de enseñanza-aprendizaje (Seoane-Pardo et all, 2006), si bien existen diferencias entre las acciones que realizan los tutores en base a los modelos instruccionales de los cursos que tutorizan, ya que, tal y como expone Gutiérrez (2008), en los modelos de aprendizaje colaborativo se requiere que el tutor medie en la construcción de conocimiento y en la creación de un ambiente en el que se perciba la dimensión social del proceso, si bien, otros autores y en el caso de los Open Online Courses, inciden en que no hay diferencias medibles entre los resultados de cursos autodidactas y sin fechas de entrega y los de duración limitada en los que los tutores intervienen y dirigen los debates (Heller, R.F, Chilolo, E., Elliott, J., Johnson, B., Lipman, D. y Richards, J., 2019) con lo que se requieren de estudios comparativos y experimentales que aborden la cuestión con el fin de dirimir hasta qué punto la intervención tutorial mejora los resultados de aprendizaje y la satisfacción percibida de los estudiantes pues, entre unos modelos y otros, la dedicación y esfuerzo de los tutores es sustancialmente diferente, y por lo tanto, también los costes del proceso.

En el estudio realizado, al comparar los datos por modelo tutorial seguido, se ha podido observar que la distribución de los datos en las encuestas ha sido asimétrica, concentrándose la mayor frecuencia de respuestas en las puntuaciones más altas "Más bien de acuerdo" (4) y "Totalmente de acuerdo" (5), si bien hay que destacar que en el modelo reactivo las puntuaciones "Totalmente en desacuerdo" (1), "Más bien en desacuerdo" (2) y "Término medio" (3), son mayores que en los cursos de tutorización 
proactiva, confirmándose diferencias estadísticamente significativas en lo que respecta a la variable Organización y Satisfacción.

Las puntuaciones de la variable Tutor, no presentan diferencias estadísticamente significativas entre un modelo tutorial y otro, posiblemente porque el mismo equipo de docentes trabaja indistintamente en unos y otros cursos. Esta cuestión pone de manifiesto la importancia de que los tutores tengan una extensa experiencia docente, formación en e-learning (Romero-Rodríguez, 2017) y desarrollen un conjunto de competencias en inteligencia emocional, altamente valoradas por los alumnos, tales como: la empatía, la orientación al servicio y la comunicación; que son claves en la efectividad de los tutores (Youde, 2016).

En base al análisis realizado, se puede concluir que, el nivel de satisfacción de los estudiantes es mayor en los cursos que siguen un modelo tutorial proactivo que en los que siguen un modelo reactivo, confirmando un trabajo de investigación previo de casos estudio (Vercher-Ferrándiz, 2019). La diferencia entre un modelo y otro está en que los tutores, además de seguir las pautas generales de comunicación e instruccionales, están en todo momento pendientes del avance del alumnado, generando interrelaciones significativas a partir del seguimiento y acompañamiento continuado y del diálogo frecuente (Youde, 2019).

También se confirma, en el contexto analizado que, un modelo de tutorización proactiva, propicia un mayor porcentaje de aptos, tras la evaluación de adquisición de conocimientos final, y aumenta la tasa de respuesta a las encuestas, siendo menor, el número de respuestas en blanco que en los cursos que siguen un modelo reactivo.

$\mathrm{Al}$ analizar los datos en base a la duración de los cursos, se confirman diferencias estadísticamente significativas a nivel global entre un modelo y otro, si bien al analizar los pares de combinaciones posibles entre modelos y duración no existe un patrón claro que permita concluir que la duración de un curso incide de forma clara en nivel de satisfacción.

Al respecto de las diferencia entre el porcentaje de aptos por modelo y duración, la diferencia menor se evidencia en los curso más cortos, cuyo temario es menos exigente, siendo mayor en los más largos, dónde además, la tasa de respuesta es mayor.

Con todo ello, se puede confirmar que, en los cursos estudiados de formación continua online del área de finanzas del Centro de Formación Permanente de la UPV, un modelo de tutorización online, en el que el tutor es proactivo y se implica en el seguimiento continuado y la evolución de sus alumnos, obtiene mejores resultados en lo que respecta al nivel de satisfacción y al porcentaje de aptos, siendo la diferencia en el porcentaje de aprobados más evidente en los cursos más exigentes y de mayor duración, dónde los estudiantes requieren de un mayor esfuerzo y voluntad para enfrentarse a los obstáculos y a las distracciones (Broc Cavero, 2011).

A pesar del elevado número de datos, no se puede obviar que este estudio se circunscribe a un área concreta de conocimientos y a un único modelo instruccional, siendo el mismo equipo de docentes el que realiza las labores de tutorización en unos y otros cursos, lo que representa una limitación al estudio realizado.

Es de interés futuro poder contrastar los resultados obtenidos en otras áreas en las que se sigan modelos pedagógicos diferentes y a partir de una selección muestral que ofrezca garantías de representatividad de la población a estudiar ya que son muchos 
los trabajos que describen y afirman la importancia del apoyo sistemático y organizado de los tutores online (Fernández-Jiménez, 2015; Seoane-Pardo et al., 2006; Yot Domínguez y Marcelo García, 2013) entre otros pero, hay pocos estudios empíricos confirmatorios de este hecho.

\section{Referencias}

Borges, F. (2005). The frustrations of online students. Causes and preventative actions. Digithum, 7, 1-9. doi: 10.7238/d.v0i7.536

Broc Cavero, M. Á. (2011). Voluntad para estudiar, regulación del esfuerzo, gestión eficaz del tiempo y rendimiento académico en alumnos universitarios. Revista de Investigación Educativa, 29(1), 171-185. Recuerado de https://revistas.um.es/rie/article/ view/110731

Cabero-Almenara, J. (2004). La función tutorial en la teleformación. En F. Martínez Sánchez \& M. P. Prendes Espinosa (Coord.). Nuevas tecnologías y educación (pp. 129144). Madrid: Pearson Educación.

Castañeda, L., Gutiérrez-Porlán, I., Prendes-Espinosa, M. P., \& Sánchez-Vera, M. del M. (2017). Formación docente para la enseñanza en línea en el contexto de la Formación Profesional. Universitas tarraconensis: Revista de ciències de l'educació, 2, 29-44. doi: 10.17345/ute.2017.2

Cohen, L., Manion, L., \& Morrison, K. (2017). Research methods in education (8. ${ }^{\mathrm{a}}$ ed.). Abingdon, Reino Unido: Routledge.

Fernández-Jiménez, M. Á. (2015). Tutoría en e-learning. Funciones y roles del tutor en la formación online. (Tesis doctorado, Universidad de Málaga). Recuperado de http:// hdl.handle.net/10630/11512

Fernández-Jiménez, M. Á., Mena-Rodríguez, E., \& Tójar-Hurtado, J. C. (2017). Funciones de la tutoría en e-learning: Estudio mixto de los roles del tutor online. Revista de Investigacion Educativa, 35(2), 409-426. doi: 10.6018/rie.35.2.273271

Fernández Navas, M. (2016). Estudio de un caso de buenas prácticas de enseñanza virtual en educación superior: El PPSC. Revista de Formación e Innovación Educativa Universitaria, 9(1), 50-66. Recuperado de http://refiedu.webs.uvigo.es/num_es.htm

Fernández Robles, B., \& Cabero Almenara, J. (2016). Percepciones de teleformadores del Instituto de Formación y Estudios Sociales de Sevilla sobre la teleformación. Innoeduca. International Journal of Technology and Educational Innovation, 2(1), 4-12. doi: 10.20548/innoeduca.2016.v2i1.1218

Franco Moreno, Y. M. (2017). Rol del tutor en el contexto del aprendizaje virtual. Revista Scientific, 2(6), 270-285. doi: 10.29394/scientific.issn.2542-2987.2017.2.6.14.270-285

Gómez Gutiérrez, J. (2008). Factores críticos del e-learning: diseño y tutorización de procesos de enseñanza-aprendizaje colaborativos. Cuadernos de trabajo social, 21, 263-283. Recuperado de https://revistas.ucm.es/index.php/CUTS/article/view/ CUTS0808110263A

Heller, R. F., Chilolo, E., Elliott, J., Johnson, B., Lipman, D., Ononeze, V., \& Richards, J. (2019). Do tutors make a difference in online learning? A comparative study in two Open Online Courses. Open Praxis, 11(3), 229. doi: 10.5944/openpraxis.11.3.960 
Llorente-Cejudo, M. C. (2006). El tutor en E-learning: aspectos a tener en cuenta. Edutec: Revista electrónica de tecnología educativa, 20, 9. doi: 10.21556/edutec.2006.20.517

López Martínez, A. M. (2009). Modelo de evaluación continua formativa-formadora- reguladora y tutorización continua con soporte multimedia. (Tesis doctorado. UNED) Recuperado de http://e-spacio.uned.es/fez/view/tesisuned:Educacion-Amlopez

Martínez, M. T., \& Briones, E. M. (2007). Contigo en la distancia: la práctica tutorial en entornos formativos virtuales. Píxel-Bit. Revista de Medios y Educación, 29, 81-86. Recuperado de https://recyt.fecyt.es/index.php/pixel/article/view/61315

Pagano, C. M. (2008). Los tutores en la educación a distancia . Un aporte teórico. Revista de Universidad y Sociedad del Conocimiento RUSC, 4(2), 1-11. doi: 10.7238/rusc.v4i2.304

Rodríguez Fernández, N. (2014). Fundamentos del proceso educativo a distancia: enseñanza, aprendizaje y evaluación. RIED. Revista Iberoamericana de Educación a Distancia, 17(2), 75-93. doi: 10.5944/ried.17.2.12679

Romero-Rodríguez, J. (2017). Representación del conocimiento experto de un tutor e-learning a través del mapa conceptual: Un modelo de buenas prácticas docentes. (Trabajo Final Grado. Universidad de Granada). Recuperado de https://digibug.ugr.es/handle/10481/46413

Rovai, A. P., \& Downey, J. R. (2010). Why some distance education programs fail while others succeed in a global environment. Internet and Higher Education, 13(3), 141-147. doi: 10.1016/j.iheduc.2009.07.001

Sagastume, F., Morales, M., Amado, H., \& Hernández, R. (2018). La importancia del tutor en los cursos virtuales: experiencia, buenas prácticas y recomendaciones. En Proceedings of the Digital Worl Learning Conference (pp. 91-97). Recuperado de http:// www.galileo.edu/page/wp-content/uploads/2019/02/index.html

Seoane-Pardo, A. M. (2014). Formalización de un modelo de formación online basado en el factor humano y la presencia docente mediante un lenguaje de patrón. (Tesis doctorado. Universidad de Salamanca). doi: 10.14201/gredos.123342

Seoane-Pardo, A. M., García-Peñalvo, F. J., Nieto-Bosom, A., Fernández-Recio, E., \& Hernández-Tovar, M. J. (2006). Tutoring on-line as quality guarantee on elearningbased lifelong learning. Definition, modalities, methodology, competences and skills. En CEUR Workshop Proceedings (Vol. 186, pp. 41-55). Recuperado de https://gredos. usal.es/handle/10366/123197

Sherry, L. (1995). Issues in distance learning. International journal of educational telecommunications, 1(4), 337-365. Recuperado de https://www.learntechlib.org/primary/p/8937

UNESCO (2015). Declaración de Qingdao. Conferencia internacional sobre las tecnologías de la información y la comunicación (TIC) y la educación. Recuperado de https://unesdoc.unesco.org/ark:/48223/pf0000233352

Vercher-Ferrándiz, M. L. (2019). La gestión de la inteligencia emocional como competencia distintiva en la docencia online para la mejora de la gestión del proceso enseñanza-aprendizaje en el ámbito de las ciencias sociales. (Tesis Doctorado. Universitat Politècnica de València). doi: 10.4995/Thesis/10251/124817

Vlachopoulos, D., \& Makri, A. (2019). Online communication and interaction in distance higher education: A framework study of good practice. International Review of Education, 65(4), 605-632. doi: 10.1007/s11159-019-09792-3 
Yot Domínguez, C., \& Marcelo García, C. (2013). Tareas y competencias del tutor online. Profesorado, 17(2), 305-325. Recuperado de https://recyt.fecyt.es/index.php/ profesorado/issue/view/2343

Youde, A. (2019). A Conceptual Framework for Effective Tutors and Tutoring within a Blended Learning Context. En EduLearn19: 11th International Conference on Education and New Learning Technologies Proceedings (pp. 317-323). doi: 10.21125/edulearn.2019.0118

Youde, Andrew. (2016). Tutor Emotional Competences Valued by Learners in a Blended Learning Contex. European Journal of Open, Distance and E-Learning, 19(2), 63-79. doi: 10.1515/eurodl-2016-0008

Fecha de recepción: 10 de febrero de 2020.

Fecha de revisión: 29 de febrero de 2020.

Fecha de aceptación: 26 de abril de 2020. 